\title{
Development of a methodology to estimate a region and waiting period for strong Kamchatka earthquakes based on seismic and ionospheric predictive signs
}

\author{
Vadim Bogdanov ${ }^{1, *}$ and Aleksey Pavlov ${ }^{1, * *}$ \\ ${ }^{1}$ Institute of Cosmophysical Research and Radio Wave Propagation FEB RAS, Paratunka, Russia
}

\begin{abstract}
In this paper, an attempt is made to combine seismological and ionospheric predictive signs formed on the eve of earthquakes in order to develop a methodology for assessing the region and the time period of waiting for strong Kamchatka earthquakes with energy class $K_{S} \geq 13.5(M \geq 6.0)$. The seismological parameter determined on the basis of the probabilistic model of the seismicity of Kamchatka region and allowing to estimate the region and the probability of occurrence of a strong earthquake in it is proposed as a mediumterm precursor. The complex of ionospheric disturbances is considered as a short-term precursor with a waiting period of up to 5 days for earthquakes. The estimation of their prognostic efficiency for the considered precursors is carried out by methods A. A. Gusev and G. M. Molchan, and their joint analysis was performed on the eve of earthquakes with $K_{S} \geq 13.5(M \geq 6.0)$, that occurred in the period 2009-2018.
\end{abstract}

\section{Introduction}

In $[1,2]$, the results of ionospheric observations were presented for the purpose of short-term (3-5 days) assessment of the waiting period for Kamchatka earthquakes. At the same time, the application of the theoretical-probabilistic approach to the catalogue of Kamchatka earthquakes [3,4] allowed to calculate the probability of seismic events entering the seismofocal zone of the Kuril Islands and southern Kamchatka and to establish for it the growth of seismic activity (twice) that occurred during 2007-2010. This made it possible to determine the area of expectation (medium-term forecast) of major earthquakes. During the period from 2011-2013, 11 seismic events with $K_{S} \geq 14.0$ occurred in this area. There is a natural task to combine seismological and ionospheric predictive signs formed on the eve of earthquakes, to assess their prognostic efficiency in order to develop a methodology for assessing the area and the time period of waiting for strong Kamchatka earthquakes with energy class $K_{S} \geq 13.5$ $(M \geq 6.0)$. This work is devoted to the solution of this problem.

\section{Method of calculation of seismic precursor of earthquakes}

\subsection{Probabilistic model of seismic regime}

Application of A. N. Kolmogorov's axiomatics to the catalogue of seismic events allows to consider each earthquake as an elementary event $\omega_{i}$, and the whole catalogue or its separate

\footnotetext{
*e-mail: vbogd@ikir.ru

**e-mail: pavlov@ikir.ru
} 
part as a space of elementary events $\Omega$ [3, 4]. Each elementary event $\omega_{i}$ can be characterized by four random variables $\xi_{i}\left(\omega_{i}\right)=\varphi_{i}\left(\omega_{i}\right), \lambda_{i}\left(\omega_{i}\right), h_{i}\left(\omega_{i}\right), K_{i}\left(\omega_{i}\right)$, where quantities $\varphi_{i}, \lambda_{i}, h_{i}$ are the coordinates of the earthquake, and $K_{i}$ - energy class. (Event time $t_{i}\left(\omega_{i}\right)$, as having no mathematical expectation, is excluded from the system of random variables). For a set of seismic events that occurred over a period of time $\Delta T$, the probability space is a collection of three objects $\{\Omega, \tilde{F}, P\}$, where $\Omega$ - space of elementary events, $\tilde{F}$ - set of subsets of random events, $P$ - probabilities of these events. Random events (for a specific selected period of time $\Delta T$ ) can represent an arbitrary combination of a variable number of random variables (when fixing others) and form some in $\tilde{F}$ subsets $A, B, C$ and etc.

For continuous quantities that define a seismic event, the distribution density can be represented as a multidimensional derivative of the distribution $F(\varphi, \lambda, h, K)$, and as a product of conditional and unconditional functions $f(\varphi, \lambda, h, K)$. If the analytical form of the distribution density is known $f(\varphi, \lambda, h, K)$, you can calculate the probability that a seismic event will fall within the specified intervals: latitude $\Delta \varphi=\varphi_{i}-\varphi_{i-1}$, longitude $\Delta \lambda=\lambda_{j}-\lambda_{j-1}$, depth $\Delta h=h_{m}-h_{m-1}$ and energy class $\Delta K=K_{n}-K_{n-1}$

$$
\begin{array}{r}
P\left(\Delta \varphi_{i}, \Delta \lambda_{j}, \Delta h_{m}, \Delta K_{n}\right)=\int_{\varphi_{i-1}}^{\varphi_{i}} d \varphi \int_{\lambda_{j-1}}^{\lambda_{j}} d \lambda \int_{h_{m-1}}^{h_{m}} d h \int_{K_{n-1}}^{K_{n}} f(\varphi, \lambda, h, K) d K= \\
=F\left(\varphi_{i}, \lambda_{j}, h_{m}, K_{n}\right)-F\left(\varphi_{i-1}, \lambda_{j-1}, h_{m-1}, K_{n-1}\right)= \\
=P\left(\Delta \varphi_{i}\right) P\left(\Delta \lambda_{j} \mid \Delta \varphi_{i}\right) P\left(h_{m} \mid \Delta \varphi_{i}, \Delta \lambda_{j}\right) P\left(K_{n} \mid \Delta \varphi_{i}, \Delta \lambda_{j}, h_{m}\right)
\end{array}
$$

where $i, j, m$ and $n$-indices corresponding to intervals of random variables. Statistical processing of the catalog by the formula (1) makes it possible to calculate not only the average probability of occurrence of a seismic event in a given interval of geographical coordinates, depth and energy class through the corresponding conditional probabilities, but also to obtain numerical values of the step distribution function $F(\Delta \varphi, \Delta \lambda, \Delta h, \Delta K)$. As the number of events $n$ increases and the interval $\Delta$ decreases, the relative frequency $v$ tends to its mathematical counterpart $P$, and $F(\Delta \varphi, \Delta \lambda, \Delta h, \Delta K)$ - towards sustainable continuous distribution $F(\varphi, \lambda, h, K)$. When calculating the function $F$ for a given time interval $\Delta T$ in the selected volume $V$, the complex of conditions leading to the realization of a random event is averaged, and, therefore, $F$ on average describes the seismic regime. In this approach, a catalog of earthquakes for the entire instrumental observation period $T_{\text {obs }}$ presented in the form of a mathematical object with three elements $\{\Omega, \tilde{F}, P\}$, can be considered as a reference model against which the current changes in seismic activity are monitored, i.e. to register variations in the probability distribution in local areas of the seismic region due to changes in the activity of a volume for different time intervals $\Delta T\left(\Delta T<T_{\mathrm{obs}}\right)$.

A random event was defined as a random event on the basis of which the seismic predictive feature is calculated $A$ : «Hit of epicenters of seismic events that occurred in the selected volume $V$, in the specified intervals of latitude $\Delta \varphi$ and longitude $\Delta \lambda \gg$.

Let us consider the method of calculating the prognostic feature based on the probabilistic parameter $P(A)$ for the study of changes in the seismic regime before strong earthquakes with $K_{S} \geq 13.5(M \geq 6.0)$. The catalogue of earthquakes of Kamchatka Branch of Federal Research Center "United Geophysical Service RAS" [5] during 1962-2018 was used for the analysis. 


\subsection{The method of calculation of seismic parameter}

\subsubsection{The division of the seismically active area into the elementary squares}

The studied seismically active area $S$ located along the Eastern coast of Kamchatka, including the Southern and Northern seismofocal zones, is divided into $m=12$ non-overlapping areas with dimensions of $S_{i}=150 \times 150 \mathrm{~km}$ (Fig. 1). Since seismic events with energy class $K_{S}^{\mathrm{pr}} \geq 13.5$ are considered as predicted earthquakes in this paper, consequently, according to [6] the length of the gap in the focus of such earthquakes is $L \geq 11 \mathrm{~km}$. Assuming that the linear size of the earthquake preparation area is of the order of 10 rupture lengths, i.e. $\sim 110$ $\mathrm{km}$, the spatial cell size of $150 \times 150 \mathrm{~km}$ can be chosen as the base case for calculations.

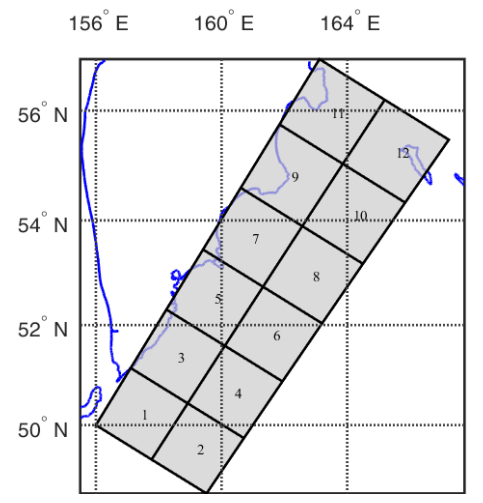

Figure 1. Division of the studied seismic area $S$ into unit cells $S_{i}$ with dimensions of $150 \times 150 \mathrm{~km}$.

\subsubsection{Calculation of the probability of seismic events in the region of the partition}

For each square $S_{i}(i=\overline{1, m})$ in a moving time window $\Delta T_{k}$ passing with step $\Delta t$, the time interval $T$ calculates the probability of seismic events $P_{k}\left(S_{i}\right)=n_{i k} / N_{k}$, where $n_{i k}$ - number of seismic events with energy class $K_{S} \geq 9.0$, occurred in the area of $S_{i}$ for the period $\Delta T_{k}, N_{k}=\sum_{k=1}^{m} n_{i k}$ - total number of earthquakes with $K_{S} \geq 9.0$, took place on the square $S=\sum_{i=1}^{m} S_{i}$ during $\Delta T_{k}$. Also in each region $S_{i}$ long-term (background) values of probability of seismic events hit are calculated $P_{T}\left(S_{i}\right)=n_{i T} / N_{T}$, where $n_{i T}$ - number of seismic events with energy class $K_{S} \geq 9.0$, occured during $T, N_{T}=\sum_{k=1}^{m} n_{T k}$ - total number of earthquakes with $K_{S} \geq 9.0$, occurred during $T$ on the square $S$. In this paper, the size of the time window and its offset step are set equal, respectively $\Delta T_{k}=1$ year and $\Delta t=1$ day, a time interval at which the study of the seismic regime is equal to $T=01.01 .1962-01.01 .2019$. On Fig. 2, as an example, the values of probabilities of seismic events in the region $S_{12}$ are presented.

\subsubsection{Calculation of statistically significant deviations of probabilities from background values}

To obtain statistically significant deviations of the current probability values $P_{k}\left(S_{i}\right)$ from their long-term (background) values $P_{T}\left(S_{i}\right)$ the parameter $\xi_{P}$, is used, determined on the basis of [7] and presented as:

$$
\xi_{P}= \begin{cases}\frac{\Delta P_{k}-\operatorname{sign}\left(\Delta P_{k}\right) \cdot\left|\sigma_{P_{k}}\right|}{\left|\sigma_{P_{T}}\right|}, & \text { if }\left|\Delta P_{k}\right|>\left|\sigma_{P_{k}}\right| \\ 0, & \text { if } 0 \leq\left|\Delta P_{k}\right| \leq\left|\sigma_{P_{k}}\right|\end{cases}
$$




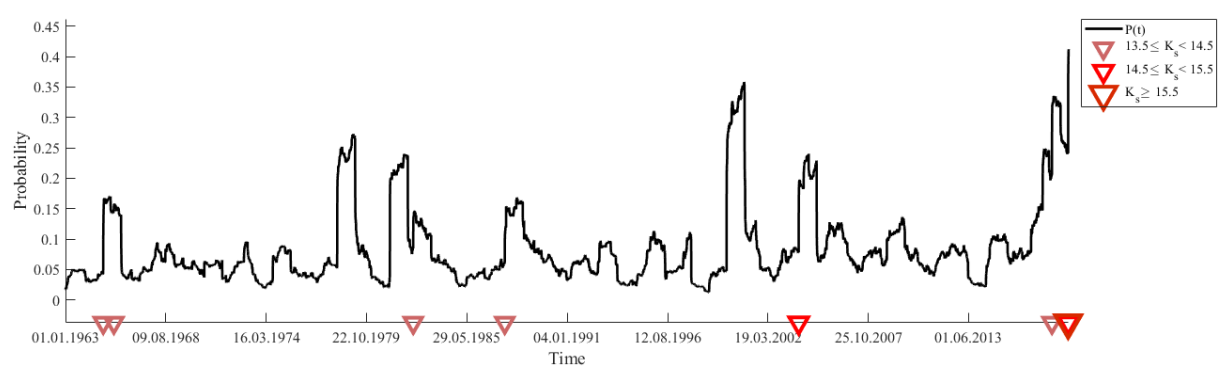

Figure 2. Time series of probability values $P_{k}\left(S_{12}\right)$.

where $P_{k}$ and $\sigma_{P_{k}}=\sqrt{\left(P_{k}\left(1-P_{k}\right) / n_{k}\right.}$ current probability values and its standard deviation in the sliding time window $\Delta T_{k} ; P_{T}$ and $\sigma_{P_{T}}=\sqrt{P_{T}\left(1-P_{T}\right) / n_{T}}$ long-term probability values and standard deviation $T>\Delta T_{k} ; n_{i}$ and $n_{T}$ - the number of seismic events that occurred over time periods, respectively $\Delta T_{k}$ and $T ; \Delta P_{k}=P_{k}-P_{T}$ the difference between the current $P_{k}$ probability value and the long-term probability value $P_{T}$.

\subsubsection{The definition of the criteria values to identify anomalies}

Strong earthquakes can be preceded by periods of increased seismic activity (an increase in the number of background events), and periods of weakening of seismic activity (a decrease in the number of background events). When you search for periods of enhanced seismicity in the beginning of the anomaly in the parameter $\xi_{P}$ is taken as the time of appearance of the values $\xi_{P} \geq \xi_{P}^{\mathrm{a}}$, where $\xi_{P}^{\mathrm{a}}$ - anxiety level is selected experimentally. In the case of searching for periods of seismic calm, the beginning of the anomaly is identified by the moment of occurrence of the values $\xi_{P} \leq \xi_{P}^{\mathrm{c}}$, where $\xi_{P}^{\mathrm{c}}-$ anxiety level is selected experimentally. Accordingly, when $\xi_{P}^{\mathrm{c}}<\xi_{P}<\xi_{P}^{\mathrm{a}}$ it is assumed that the current probability values do not significantly deviate from the background values. In this paper, the values of the criteria for the periods of activation and lull were established respectively $\xi_{P}^{\mathrm{a}}=2$ and $\xi_{P}^{\mathrm{c}}=-2$.

\subsubsection{Calculation of average earthquake waiting time}

For each area $S_{i}$, the average waiting time for an earthquake with a predicted energy class is determined $K_{S} \geq K_{S}^{\mathrm{pr}}$ as for anomalies of seismic activity and seismic anomalies lull. If in the region $S_{i}$ during the observation period $T$ there were $N$ earthquakes with $K_{S} \geq K_{S}^{\mathrm{pr}}$, of which $n_{\mathrm{a}}$ events were preceded by anomalies of seismic activation and the waiting time from the beginning of the $j$-th anomaly to the earthquake was $T_{\mathrm{a} j}\left(j=\overline{1, n_{\mathrm{a}}}\right)$, the average waiting time for this anomaly will be $\tilde{T}_{\mathrm{a}}=\frac{1}{n_{\mathrm{a}}} \sum_{j=1}^{n_{\mathrm{a}}} T_{\mathrm{a} j}$. Similarly, the average waiting time for a seismic calm anomaly can be defined as $\tilde{T}_{\mathrm{c}}=\frac{1}{n_{\mathrm{c}}} \sum_{l=1}^{n_{\mathrm{c}}} T_{\mathrm{cl}}$, where $n_{\mathrm{c}}$ - number of earthquakes with $K_{S} \geq K_{S}^{\mathrm{pr}}$, occurred after the seismic lull; $T_{\mathrm{cl}}$ - waiting time from the beginning of the $l$-th anomaly to the earthquake $\left(l=\overline{1, n_{\mathrm{c}}}\right)$. When calculating the average waiting time, false anomalies (not completed by earthquakes) are not taken into account, and the waiting periods for anomalies of different types do not overlap. Thus, the waiting time for activation and lull anomalies, taking into account the standard deviation, can be represented as follows: $T_{\mathrm{a}}^{\mathrm{w}}=\tilde{T}_{\mathrm{a}} \pm \sigma_{T_{\mathrm{a}}}$ and $T_{\mathrm{c}}^{\mathrm{w}}=\tilde{T}_{\mathrm{c}} \pm \sigma_{T_{\mathrm{c}}}$. The average waiting period for an earthquake for all anomalies both in activation and calm: $\tilde{T}_{\mathrm{w}}=\frac{1}{n_{\mathrm{a}}+n_{\mathrm{c}}}\left(\sum_{j=1}^{n_{\mathrm{a}}} T_{\mathrm{a} j}+\sum_{l=1}^{n_{\mathrm{c}}} T_{\mathrm{cl}}\right)$. Fig. 3 presents the timing values parameter $\xi_{P}$ for region $S_{12}$ and the periods on the basis of which were calculated $T_{\mathrm{a}}^{\mathrm{w}}$ and $T_{\mathrm{c}}^{\mathrm{w}}$. 


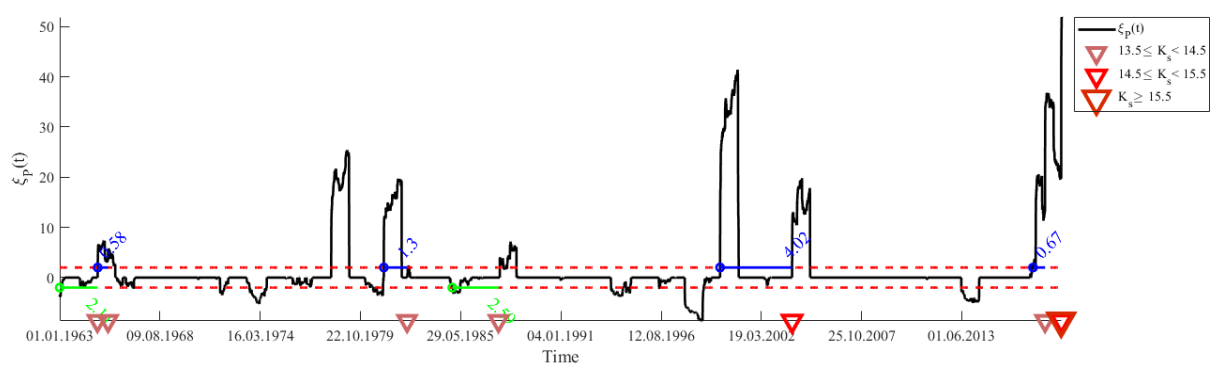

Figure 3. Temporary values of the parameter $\xi_{P}$ for region $S_{12}$. Red triangles on the time axis indicate earthquakes with $K_{S} \geq 13.5$. The blue color indicates the duration of the waiting periods earthquake $K_{S} \geq 13.5$ when identifying anomalies activation of the seismic regime, green - the duration of waiting periods for earthquakes when identifying anomalies calm seismic regime.

\subsubsection{Evaluation of the effectiveness of the prognostic feature $\xi_{P}$}

According to Sect. 2.2.5 the waiting periods of the earthquake with $K_{S} \geq K_{S}^{\mathrm{pr}}$ in each of the considered areas $S_{i}$ are assumed to be equal $T_{\mathrm{a}}^{\mathrm{w}}=\tilde{T}_{\mathrm{a}} \pm \sigma_{T_{\mathrm{a}}}$ (seismic activity) and $T_{\mathrm{c}}^{\mathrm{w}}=$ $\tilde{T}_{\mathrm{c}} \pm \sigma_{T_{\mathrm{c}}}$ (seismic calm). For each type of anomaly on the area $S_{i}$ the number of events is counted $n_{\mathrm{a}}$ and $n_{\mathrm{c}}$, that fell into the waiting periods, respectively, is calculated $T_{\mathrm{a}}^{\mathrm{w}}$ and $T_{\mathrm{c}}^{\mathrm{w}}$. If a seismic event of the predicted energy class occurred during the waiting period, the anomaly is considered realized and the earthquake is considered predicted. If a group of earthquakes occurred during the waiting period, they are treated as a single event. If no event occurs during the waiting period, the anomaly is considered false. For each type of anomaly, the total waiting time for a predicted earthquake is calculated for each area, including both waiting periods for realized anomalies and waiting periods for false anomalies. For example, if $n_{i}$ seismic events occurred in the study area during the observation period $T, z$ of which was predicted and $a$ anomalies of the seismic regime were detected, the total waiting time (alarm time) for earthquakes can be represented as: $T_{i}^{\text {alarm }}=\sum_{j=1}^{z} T_{j}^{\mathrm{pr}}+\sum_{u=1}^{a-z} T_{u}^{\mathrm{fa}}$, where $T_{j}^{\mathrm{pr}}$-duration of the period from the beginning of the anomaly to the $j$-th predicted earthquake, $T_{u}^{\mathrm{fa}}-$ the duration of the false alarm period. Based on the alarm periods $T_{i}^{\text {alarm }}$ defined for each area $S_{i}$ the average alarm time for all the considered areas is calculated: $\tilde{T}_{\text {alarm }}=\frac{1}{m} \sum_{k=1}^{m} T_{k}^{\text {alarm }}$, where $m-$ number of areas. To assess the prognostic effectiveness of a prognostic feature $\xi_{P}$ following parameters are used: reliability $R$, validity $V$, the effectiveness of A. A. Gusev and G. M. Molchan's methods. The reliability of the precursor $R$ is defined as the ratio of the number of earthquakes $n\left(E_{A}\right)$ for which the precursor was allocated to the number of all earthquakes $n(E)$ [8]:

$$
R=\frac{n\left(E_{A}\right)}{n(E)}
$$

The validity of the precursor is defined as the ratio of the number of precursor anomalies $n\left(A_{E}\right)$ to the total number of isolated anomalies $n(A)[8]$ :

$$
V=\frac{n\left(A_{E}\right)}{n(A)}
$$

The efficiency of a prognostic feature $\xi_{P}$ (separately for anomalies seismic activity or calm) on the basis of method A. A. Gusev [9] is calculated by the formula:

$$
J_{G}=\frac{n / T_{\text {alarm }}}{N / T}
$$


Table 1. Predictive efficiency of the parameter $\xi_{P}$.

\begin{tabular}{|c|c|c|c|c|}
\hline Type of seismic anomaly & $V$ & $R$ & $J_{G}$ & $J_{M}$ \\
\hline Seismic activity $\left(\xi_{P} \geq 2\right)$ & 0.52 & 0.57 & 2 & 0.29 \\
\hline Seismic calm $\left(\xi_{P} \leq 2\right)$ & 0.36 & 0.35 & 1.57 & 0.13 \\
\hline Seismic activity and calm & 0.4 & 0.83 & 1.65 & 0.54 \\
\hline
\end{tabular}

where $n=\sum_{i=1}^{m} n_{i}$ - total number of predicted earthquakes with $K_{S} \geq K_{S}^{\mathrm{pr}}, N=\sum_{i=1}^{m} N_{i}-$ total number of earthquakes with $K_{S} \geq K_{S}^{\mathrm{pr}}, T_{\text {alarm }}$ - average alarm time for all considered areas , $T$ - observation period, $m$ - number of areas. In the case of no earthquake-precursor relationship, i.e. random guessing, the efficiency of $J_{G}$ is 1 . The efficiency of a prognostic feature $\xi_{P}$ based on G. M. Molchan's method [10] is calculated by the formula:

$$
J_{M}=1-v-\tau
$$

where $v=1-n / N$ - target pass rate, $\tau=T_{\text {alarm }} / T$ - relative alarm volume.

The results of the evaluation of the predictive efficiency of parameter $\xi_{P}$ for earthquakes with $K_{S} \geq 13.5$, calculated according to equations (3-6) are presented in Table 1 and errors diagram (Fig. 4). As can be seen from Table 1, the share of anomalies of seismic regime

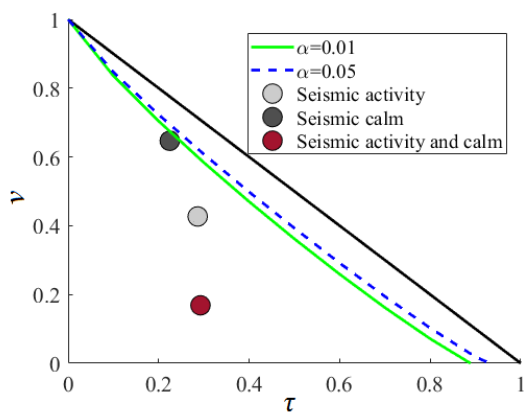

Figure 4. The diagram of errors for the parameter $\xi_{P}$ in predicting earthquakes with $K_{S} \geq 13.5$.

activation identified by parameter $\xi_{P}$ and preceding earthquakes (parameter $V$ ) was $52 \%$, and the share of calm anomalies was $35 \%$. The relative number of anomalies of the seismic regime of both types preceding the onset of earthquakes with $K_{S} \geq 13.5$, was $40 \%$. The activation of the seismic regime was preceded by $57 \%$ of earthquakes, and calm $-35 \%$. The $J_{G}$ prediction efficiency for both types of anomalies was 1.65 . Since $J_{G}>1.5$, this predictive feature is considered informative and can be used in earthquake prediction algorithms. The $J_{M}$ prediction efficiency for both types of anomalies was 0.53 and as can be seen from the error diagram (Fig. 4) the values $(\tau, v)$ lie below the lower limit of $99 \%$ of the confidence interval (green curve), which can be interpreted as a high degree of reliability of the revealed connection between the identified anomalies of the seismic regime using the parameter $\xi_{P}$ and predicted earthquakes from the considered range of the energy class.

\subsubsection{Assessment of the waiting area, time period and probability of occurrence of an earthquake with an energy class $K_{S} \geq K_{S}^{\text {pr }}$}

Let the studied seismic region be divided into $m$ elementary cells $S_{i}$, where $i=\overline{1, m}$, which form the area of $S$. Area of expectations of strong earthquake will be considered $S_{\exp }$, 
which includes the unit cells, which was identified anomalies of seismic regime parameter $\xi_{P}: S_{\exp }=\sum_{j=1}^{l} S_{j}$, where $l \leq m-$ number of areas where abnormal parameter values are identified $\xi_{P}$.

The average waiting period for a strong earthquake will be considered the period $\tilde{T}_{\mathrm{w}}=$ $\frac{1}{l} \sum_{j=1}^{l} T_{\mathrm{w} j}$, where $l$ - number of areas where abnormal parameter values are identified $\xi_{P}$, $T_{\mathrm{w} j}=\tilde{T}_{\mathrm{w} j}+\sigma_{T}-$ the waiting period earthquake in the cell $S_{j}, j=\overline{1, l}$.

Probability of occurrence earthquake with $K_{S} \geq K_{S}^{\mathrm{pr}}$ in the range $S_{\text {exp }}$, it was calculated on the basis of Bayes formula, similar to the approach taken in [7]. Let $D_{1}$ event - an earthquake occurrence predicted class $K_{S} \geq K_{S}^{\mathrm{pr}}$ in the range $S_{\text {exp. }}$. The probability of this event is defined as $P\left(D_{1}\right)=\frac{n_{l}}{N}$, where $n_{l}=\sum_{j=1}^{l} n_{j}$ - number earthquakes with $K_{S} \geq K_{S}^{\mathrm{pr}}$, occurred in the area of $S_{\text {exp }}$ during the period of observation $T, N$ - number earthquakes with $K_{S} \geq K_{S}^{\mathrm{pr}}$, occurred in the area of $S$ during the period of observation. Then the event $D_{2}-$ absence earthquake with $K_{S} \geq K_{S}^{\mathrm{pr}}$ in the range $S_{\text {exp }}$, the probability of which $P\left(D_{2}\right)=1-P\left(D_{1}\right)$.

Let event $E$ is the occurrence of anomalous values of the parameter $\xi_{P}$. Then the event $E$ can be represented in the form:

$$
E=D_{1} E+D_{2} E
$$

Event $D_{1} E$ - earthquake occurrence is represented as: with $K_{S} \geq K_{S}^{\mathrm{pr}}$ in the range $S_{\text {exp }}$ when identifying anomalies of the seismic regime (activation or calm). The probability of this event is defined as $P\left(D_{1} E\right)=P\left(D_{1}\right) \cdot P\left(E \mid D_{1}\right)$, where $P\left(E \mid D_{1}\right)=\frac{n_{\mathrm{pr}}}{N_{S_{\mathrm{exp}}}}-$ probability of occurrence of a seismic event in the identification of anomalies of the seismic regime in the region $S_{\text {exp }} ; n_{\mathrm{pr}}$ - number of events with $K_{S} \geq K_{S}^{\mathrm{pr}}$, predicted when an anomaly appears during the observation period $T ; N_{S_{\text {exp }}}$ - total number of events with $K_{S} \geq K_{S}^{\mathrm{pr}}$, what happened in the area $S_{\exp }$ over the period $T$.

Event $D_{2} E$ - the lack of earthquakes with $K_{S} \geq K_{S}^{\mathrm{pr}}$ in the area $S_{\exp }$ when identifying anomalies of the seismic regime (activation or calm). The probability of this event is defined as $P\left(D_{2} E\right)=P\left(D_{2}\right) \cdot P\left(E \mid D_{2}\right)$, where $P\left(E \mid D_{2}\right)=\frac{n_{\mathrm{fa}}}{N_{\mathrm{ta}}}$ - probability of occurrence of abnormal parameter values $\xi_{P}$ (false alarms) in the area $S_{\text {exp }}$, which did not end with earthquakes; $n_{\mathrm{fa}}-$ number of false alarms per observation period $T ; N_{\mathrm{ta}}$ - the total number of cases of abnormal behavior of the parameter $\xi_{P}$ in the area $S_{\text {exp }}$ over the period $T$.

Probability of occurrence of a seismic event with $K_{S} \geq K_{S}^{\mathrm{pr}}$ in the area $S_{\text {exp }}$ (random event $D_{1}$ ), provided that the anomaly of the seismic regime is identified and the alarm mode is declared (random event $E$ ), it is calculated on the basis of the Bayes formula:

$$
P\left(D_{1} \mid E\right)=\frac{P\left(D_{1}\right) \cdot P\left(E \mid D_{1}\right)}{P\left(D_{1}\right) \cdot P\left(E \mid D_{1}\right)+P\left(D_{2}\right) \cdot P\left(E \mid D_{2}\right)}
$$

As an example, in Fig. 5 presents a map of expectation earthquakes with the energy class $K_{S} \geq 13.5$, built at the beginning of the day 28.02.2013. Color on the map marked the area in which anomalies were identified seismic regime based on the parameter $\xi_{P}$ and which form the waiting area of the seismic event. According to the formula (8) the probability of an earthquake with $K_{S} \geq 13.5$ in the area $S_{\exp }$ was $P\left(D_{1} \mid E\right)=0.7$, the waiting period was $T_{\mathrm{w}}=4.1 \pm 3.2$. In the area $S_{1}$ within the area $S_{\text {exp }}, 28.02 .201314: 05: 48$ at a depth of $h=61 \mathrm{~km}$ occurred seismic event energy class $K_{S}=15.2$. Fig. 6a presents the time series of probabilities $P\left(D_{1} \mid E\right)$ calculated by the formula (8) in the sliding time window $\Delta T_{k}=1$ year, passing step $\Delta t=1$ day time interval 2009-2018. Fig. 6b shows the relative number of areas in which seismic anomalies have been identified in the standby earthquake regime. Fig. $6 \mathrm{c}$ presents waiting periods $\tilde{T}_{\mathrm{w}} \pm \sigma_{T}$ earthquakes $K_{S} \geq 13.5$ in the waiting area $S_{\exp }$.

During the observation period 2009-2018, 26 earthquakes with $K_{S} \geq 13.5$ occurred in the considered seismically active region, at the same time in the waiting area $S_{\exp }$, defined 


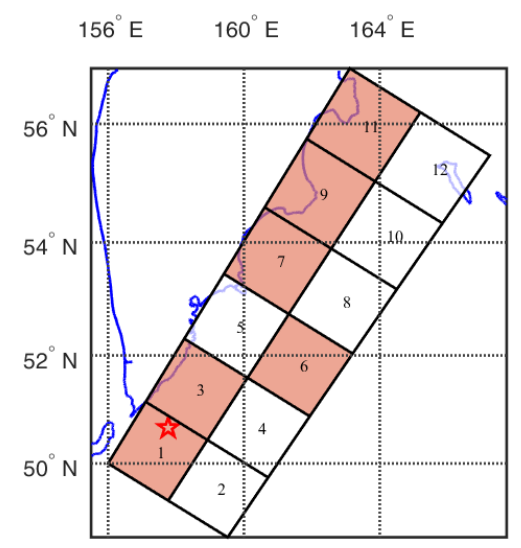

Figure 5. Map of expectation earthquakes with the energy class $K_{S} \geq 13.5$, calculated at the beginning of the day 28.02.2013.

based on parameter $\xi_{P} 21$ seismic events of the considered range of energies got. Average probability $P\left(D_{1} \mid E\right)$ of an earthquake was $\approx 0.78$.

As a seismological parameter $\xi_{P}$ is a medium-term precursor, to clarify the period of occurrence of the predicted earthquake, short-term ionospheric precursors with a waiting period of up to several days were involved.

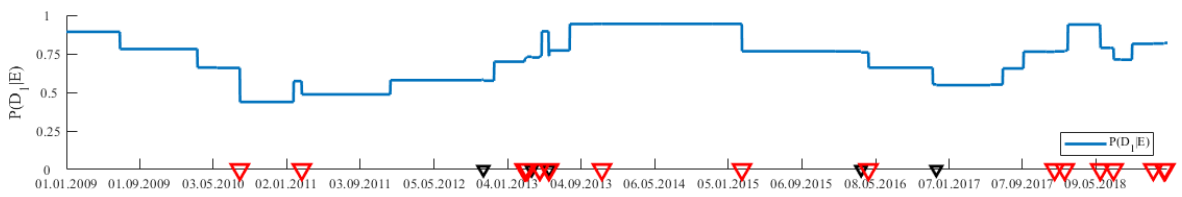

a)

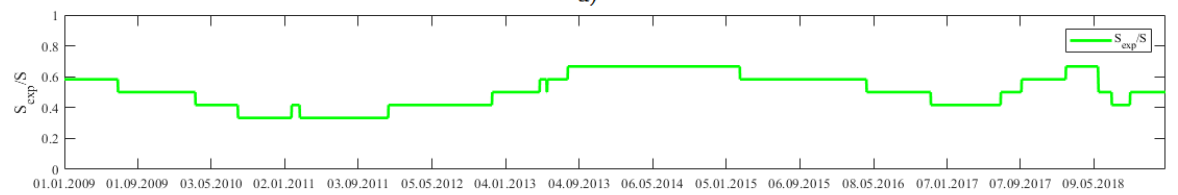

b)

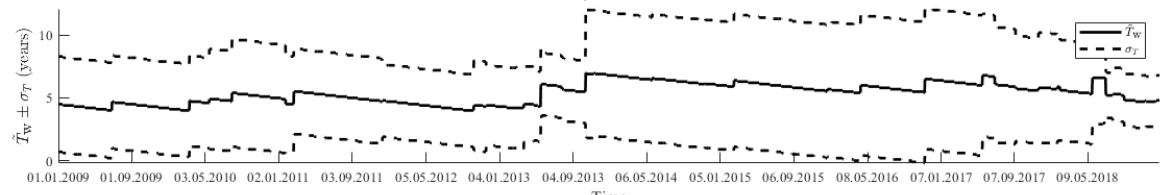

c) ${ }^{\text {Time }}$

Figure 6. (a) Time series of probabilities $P\left(D_{1} \mid E\right)$, calculated in a sliding time window $\Delta T_{k}=1$ year, passing step $\Delta t=1$ day time interval 2009-2018. Red triangles on the time axis indicate earthquakes with $K_{S} \geq 13.5$ that occurred in the waiting area for earthquakes $S_{\text {exp }}$, black triangles - earthquakes that occurred outside the waiting area $S_{\text {exp }}$; b) the relative number of regions that were in standby earthquakes $S_{\exp } / S ;$ c) waiting periods $\tilde{T}_{\mathrm{w}} \pm \sigma_{T}$. 


\section{Analysis of ionospheric precursors}

\subsection{Ionospheric precursors of earthquakes in Kamchatka region}

Modern studies have found that the earth's crust influences the physical processes occurring in the upper geospheric shells. Consequently, in seismically active regions, any anomalous changes in the behavior of ionospheric parameters, formed against the background of regular daily changes in the ionospheric characteristics due to the influence of the Sun, can provide information about the processes of earthquake preparation [11-14]. In turn, each seismically active region is characterized by its own, the most informative features (anomalies) in the behavior of the ionosphere parameters, which can be identified with the precursors of earthquakes. In the Kamchatka region, such features, as shown by long-term studies of the dynamics of ionospheric parameters on the eve of the earthquake, include the following $[1,2,15,16]$ :

1. precipitation from several hours to several days before the earthquake of charged particles from the radiation belts into the ionosphere (formation of the K-layer);

2. formation of sporadic layer Es type r 1-5 days before the earthquake;

3. on the background of the development of the magnetic storm (in spring and autumn) the anomalous increase of the critical frequency $f \mathrm{oF} 2$ (the increase in electron concentration) exceeding the median (in the ordinary course of a magnetic storm in the ionosphere due to the vortex electric field drift occurs, which leads to a shift of electrons at high altitudes and decrease their concentration).

4. formation of a diffusion layer F2 (F2-spread) lasting several hours in 1-3 days against the background of a quiet magnetosphere;

5. for 1-3 days, the stratification of the F2 layer in height and frequency (modes " $\mathrm{H}$ " and "V"), the so-called moving ionospheric disturbances.

As an example, in Fig. 7 presented ionograms containing anomalies corresponding to features 2) (Fig. 7a) and 5) (Fig. 7b).

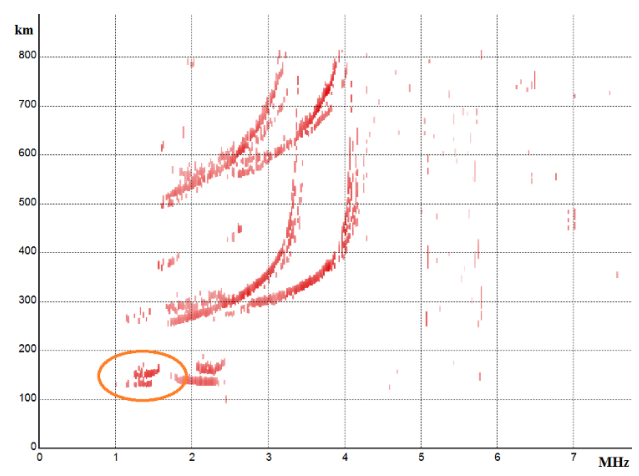

a)

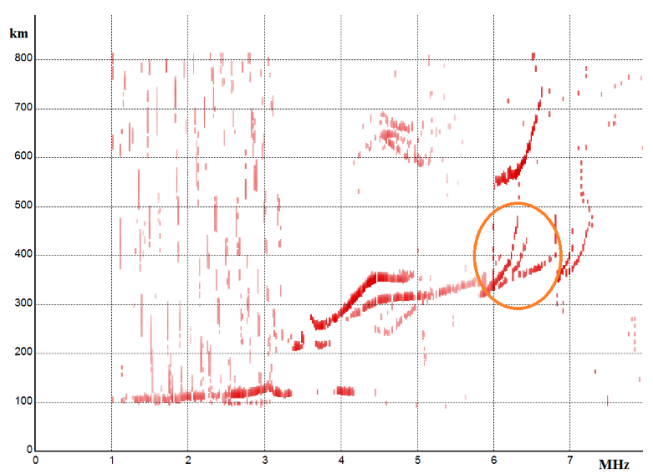

b)

Figure 7. Examples of anomalous behavior of ionospheric parameters: a) sporadic layer Es of type r; b) stratification of layer F2 in frequency (mode "V", moving ionospheric disturbances).

In this work the data of radiophysical observations made by means of vertical radiosonding are used. The automatic ionospheric station (AIS) of vertical radiosonding is located in 
Table 2. The predictive performance of each ionospheric perturbations for earthquakes with $K_{S} \geq 13.5$.

\begin{tabular}{|c|l|l|l|l|l|l|l|}
\hline $\begin{array}{c}\text { Ion. dis- } \\
\text { turbance }\end{array}$ & $\begin{array}{c}\mathrm{K}- \\
\text { layer }\end{array}$ & $\begin{array}{c}\text { Es- } \\
\text { spread }\end{array}$ & $\begin{array}{c}\text { Es } \\
\text { type } \mathrm{r}\end{array}$ & $f_{\mathrm{oF} 2}$ & $\begin{array}{c}\text { F2- } \\
\text { spread }\end{array}$ & $\begin{array}{c}\text { stratification } \\
\text { of layer F2 } \\
\text { (mode "H") }\end{array}$ & $\begin{array}{l}\text { stratification } \\
\text { of layer F2 } \\
\text { (mode "V") }\end{array}$ \\
\hline$n\left(E_{A}\right)$ & 11 & 9 & 13 & 9 & 18 & 13 & 14 \\
\hline$n(E)$ & 17 & 20 & 18 & 17 & 19 & 17 & 17 \\
\hline$n\left(A_{E}\right)$ & 11 & 9 & 13 & 9 & 18 & 13 & 14 \\
\hline$n(A)$ & 86 & 47 & 72 & 68 & 63 & 86 & 70 \\
\hline$V$ & 0.13 & 0.19 & 0.18 & 0.13 & 0.29 & 0.15 & 0.2 \\
\hline$R$ & 0.65 & 0.45 & 0.72 & 0.53 & 0.95 & 0.76 & 0.82 \\
\hline$J_{G}$ & 1.25 & 1.12 & 1.27 & 1.52 & 0.99 & 1.15 & 1.47 \\
\hline$J_{M}$ & 0.13 & 0.05 & 0.15 & 0.18 & -0.01 & 0.1 & 0.26 \\
\hline
\end{tabular}

the village of Paratunka $\left(\varphi=52.97^{\circ} \mathrm{N}, \lambda=158.25^{\circ} \mathrm{E}\right)$. Observations are made once every 15 minutes in pulse mode at frequencies from 1 to $15 \mathrm{MHz}$.

In order to select the most effective ionospheric precursors, the parameters $V, R, J_{G}, J_{M}$ were calculated using the formulas (4-6) for seismic events of the predicted energy class $K_{S} \geq$ $13.5(M \geq 6.0)$, occurred at depths up to $100 \mathrm{~km}$ at distances up to $r=500 \mathrm{~km}$ from the point of ionospheric observations of ICIR Feb RAS in Kamchatka. The observation period was chosen equal to 2013-2018 (spring and autumn seasons), the waiting period for earthquakes with $K_{S} \geq 13.5$ was set to equal $T_{\mathrm{w}}=5$ day. As can be seen from the results presented in Table 2, the most informative, i.e. with the highest values of the parameters $J_{G}$ and $J_{M}$, the following ionospheric precursors are: the critical frequency $f_{\mathrm{oF}} 2$ of the ionospheric layer $\mathrm{F} 2$, the K-layer, the F2 stratification in frequency (mode "V") and the sporadic layer Es of type $\mathrm{r}$. Since separately these ionospheric precursors do not have sufficient prognostic efficiency, the task of developing a short-term earthquake prediction algorithm based on the joint analysis of these ionospheric disturbances was set.

\subsection{Method of short-term earthquake prediction based on the complex of ionospheric precursors}

On the basis of the selected most effective ionospheric precursors, an algorithm for shortterm earthquake prediction was constructed, in which a joint analysis of the ionospheric disturbances under consideration is carried out in a sliding time window of width $\Delta T=5$ days with a step $\Delta t=1$ day. The condition for announcing the start of the waiting period To seismic event was the execution in a time interval $\Delta T$ for at least three of the four ionospheric parameters consider the following criteria:

- Exceeding the critical frequency $f$ oF2 layer F2 median values $f_{\text {med }}$, calculated for the previous 30 days, not less than $20 \%\left(\frac{f \mathrm{oF} 2-f_{\text {med }}}{f_{\text {med }}} \geq 0.2\right)$ against the background of the development of the magnetospheric storm (total values of the K-index per day $\Sigma K \geq 20$ );

- Stratification of the F2 layer in frequency (mode "V") for at least one day interval $\Delta T$;

- K-layer formation during at least one day of $\Delta T$ interval;

- Formation of sporadic layer Es type $\mathrm{r}$ for at least one day interval $\Delta T$.

The waiting period is chosen equal to $T_{\mathrm{w}}=5$ days. The algorithm was evaluated its predictive effectiveness in a time interval 2013-2018 in spring and autumn seasons for seismic 
Table 3. The predictive efficiency of the complex ionospheric precursors.

\begin{tabular}{|c|c|c|c|c|c|c|c|c|c|}
\hline$n\left(E_{A}\right)$ & $n(E)$ & $n\left(A_{E}\right)$ & $n(A)$ & $V$ & $R$ & $J_{G}$ & $\tau$ & $v$ & $J_{M}$ \\
\hline 13 & 17 & 13 & 78 & 0.17 & 0.76 & 2.01 & 0.38 & 0.24 & 0.38 \\
\hline
\end{tabular}

events at depths of $100 \mathrm{~km}$ in radius $r=500 \mathrm{~km}$ from the registration point of the ionospheric observations. The results of the evaluation are presented in Table 3 and Fig. 8.

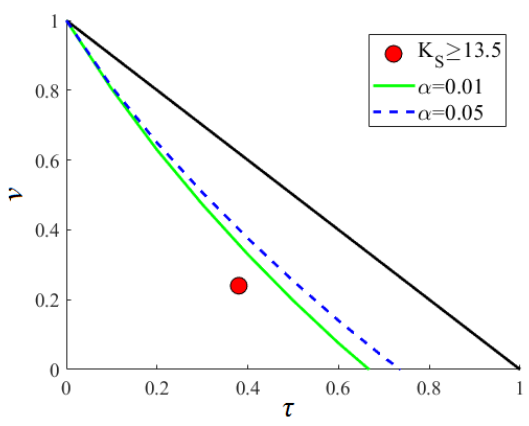

Figure 8. The chart of errors for the algorithm of the complex ionospheric precursors for $K_{S} \geq 13.5$. The lower bounds of the confidence interval of the random forecast with the significance level are marked $\alpha=0.01$ and $\alpha=0.05$.

According to the results presented in Table 3, when predicting earthquakes with energy class $K_{S} \geq 13.5$, the reliability is 0.76 (i.e. $76 \%$ of earthquakes had a precursor), and the reliability is 0.17 (i.e. $17 \%$ of detected anomalies were realized). The efficiency value $J_{G}=2.01$ shows that the prognosis by this method is statistically significant and 2 times different from random guessing. In the error diagram (Fig. 8) the values $(\tau, v)$ obtained for the range $K_{S} \geq 13.5$ lie below the lower limit of $99 \%$ of the confidence interval, which can be interpreted as a high degree of reliability of the revealed connection of the considered complex of ionospheric precursors with earthquakes of this range of energy class that occurred at distances up to $500 \mathrm{~km}$ from the observation point.

\section{Joint analysis of seismological and ionospheric precursors}

We will conduct a retrospective analysis of the behavior of the precursors considered above before the onset of strong earthquakes. Table 4 presents a list of 25 seismic events and their groups with energy class $K_{S} \geq 13.5$ that occurred during the period 2009-2018 at depths up to $100 \mathrm{~km}$ within the region $S$ (Fig. 1), but not more than $500 \mathrm{~km}$ from the point of ionospheric observations (if during one day there were several earthquakes within one unit cell $S_{i}$, they were considered as one event). For each earthquake, at the beginning of the day in which it occurred, based on the analysis of the parameter values $\xi_{P}$ determined waiting area $S_{\exp }$ and calculated the probability $P\left(D_{1} \mid E\right)$ its occurrence in the waiting area. Table 4 presents the probability values $P\left(D_{1} \mid E\right)$, and signs "+" and "-" observed earthquake respectively inside the waiting area $S_{\exp }$ and outside its borders. All things considered seismic events 20 quakes hit the area, waiting $S_{\exp }$, and 5 events in the area where the seismic activity did not exceed the background level (pass objectives). Average probability $P\left(D_{1} \mid E\right)$ the onset of an earthquake in the waiting area $S_{\exp } \sim 0.78$. Average probability $P\left(D_{1} \mid E\right)$, at which the earthquake occurred outside the waiting area $S_{\exp }$ was $\sim 0.67$. 
Table 4. List of predicted earthquakes with energy class $K_{S} \geq 13.5$ over the period 2009-2018.

\begin{tabular}{|c|c|c|c|c|c|c|c|}
\hline \multirow{2}{*}{ № } & \multirow{2}{*}{ Date EQ } & \multirow{2}{*}{ Time EQ } & \multirow{2}{*}{$K_{S}$} & \multirow{2}{*}{$S_{i}$} & \multicolumn{2}{|c|}{ Seism. prec. $\xi_{P}$} & \multirow{2}{*}{ Ion. precursors } \\
\hline & & & & & $P\left(D_{1} \mid E\right)$ & $S_{\exp }$ & \\
\hline 1 & 30.07 .2010 & $3: 56: 10$ & 14.1 & $S_{5}$ & 0.65 & + & - \\
\hline 2 & 20.02 .2011 & $21: 43: 22$ & 14.1 & $S_{11}$ & 0.57 & + & + \\
\hline 3 & 15.10 .2012 & $01: 18: 58$ & 13.5 & $S_{4}$ & 0.58 & - & + \\
\hline 4 & 28.02 .2013 & $14: 05: 48$ & 15.2 & $S_{1}$ & 0.7 & + & + \\
\hline \multirow{2}{*}{5} & 01.03 .2013 & $12: 53: 49$ & 14.2 & \multirow{2}{*}{$S_{1}$} & \multirow{2}{*}{0.7} & \multirow{2}{*}{+} & \multirow[b]{2}{*}{+} \\
\hline & 01.03 .2013 & $13: 20: 48$ & 15.1 & & & & \\
\hline 6 & 04.03 .2013 & $20: 56: 33$ & 13.6 & $S_{1}$ & 0.72 & + & + \\
\hline 7 & 09.03 .2013 & $14: 56: 27$ & 13.7 & $S_{1}$ & 0.72 & + & - \\
\hline 8 & 24.03 .2013 & $04: 18: 33$ & 13.8 & $S_{4}$ & 0.73 & - & + \\
\hline 9 & 19.04 .2013 & $19: 58: 37$ & 13.8 & $S_{1}$ & 0.72 & + & + \\
\hline 10 & 20.04 .2013 & $13: 12: 46$ & 14.8 & $S_{1}$ & 0.73 & + & + \\
\hline 11 & 19.05 .2013 & $18: 44: 07$ & 13.7 & $S_{6}$ & 0.89 & + & + \\
\hline 12 & 19.05 .2013 & $22: 40: 22$ & 13.5 & $S_{5}$ & 0.89 & + & \\
\hline 13 & 21.05 .2013 & $01: 55: 03$ & 13.6 & $S_{6}$ & 0.73 & + & \multirow{3}{*}{-} \\
\hline \multirow{2}{*}{14} & 21.05 .2013 & 03:08:16 & 13.9 & \multirow{2}{*}{$S_{5}$} & \multirow{2}{*}{0.73} & \multirow{2}{*}{ - } & \\
\hline & 21.05 .2013 & $05: 43: 16$ & 14.4 & & & & \\
\hline 15 & 12.11 .2013 & 07:03:48 & 15.3 & $S_{9}$ & 0.94 & + & + \\
\hline 16 & 19.02 .2015 & $16: 32: 45$ & 13.8 & $S_{5}$ & 0.94 & + & + \\
\hline 17 & 20.03 .2016 & $22: 50: 16$ & 14.9 & $S_{10}$ & 0.76 & - & + \\
\hline 18 & 14.04 .2016 & 03:06:07 & 13.9 & $S_{7}$ & 0.76 & + & + \\
\hline 19 & 25.11 .2016 & $07: 26: 21$ & 13.5 & $S_{7}$ & 0.55 & - & + \\
\hline 20 & 22.12 .2017 & $14: 44: 16$ & 14.2 & $S_{7}$ & 0.76 & + & + \\
\hline 21 & 23.05 .2018 & $01: 37: 44$ & 14.2 & $S_{9}$ & 0.88 & + & - \\
\hline 22 & 06.07 .2018 & $01: 40: 03$ & 14.9 & $S_{3}$ & 0.79 & + & + \\
\hline 23 & 14.11 .2018 & $21: 21: 49$ & 15.0 & $S_{9}$ & 0.81 & + & + \\
\hline 24 & 20.12 .2018 & $17: 01: 53$ & 16.1 & $S_{12}$ & 0.82 & + & + \\
\hline 25 & 24.12 .2018 & $12: 41: 18$ & 14.6 & $S_{12}$ & 0.82 & + & - \\
\hline
\end{tabular}

Number of earthquakes with $K_{S} \geq 13.5$, before the onset of which the complex of ionospheric disturbances described in Sect. 3.2 was observed was 18 (in Table 4 they are marked with "+"), while 14 of them occurred in the waiting area $S_{\text {exp }}$. Before the occurrence of five earthquakes complex ionospheric disturbances have not been identified or has come after, but in the same day that the earthquake occurred, as, for example, for the event 23.05.2018 with $K_{S}=14.2$. Thus, a joint analysis of the behavior of the precursors considered in the work before the occurrence of 25 seismic events with $K_{S} \geq 13.5$ showed that 14 of them occurred in the waiting area $S_{\text {exp }}$ with probability of occurrence $P\left(D_{1} \mid E\right) \geq 0.7$, defined based on parameter $\xi_{P}$, at the same time, a complex of ionospheric disturbances was identified in the time interval up to five days prior to the occurrence of these seismic events.

\section{Conclusion}

The paper proposes an approach that combines seismological and ionospheric prognostic features to assess the areas and time periods of occurrence of earthquakes with $K_{S} \geq 13.5$ $(M \geq 6.0)$ in the Kamchatka region. 
As seismic precursor parameter is proposed $\xi_{P}$ calculated on the basis of a probabilistic model of the catalog of Kamchatka earthquakes and allowing in the medium term to assess the scope and probability of a can be severe seismic event in this area. Analysis of predictive efficiency for earthquakes as seismic precursor parameter is proposed $\xi_{P}$ calculated on the basis of a probabilistic model of the catalog of Kamchatka earthquakes and allowing in the medium term to assess the scope and probability of a can be severe seismic event in this area. Analysis of prognostic efficiency for earthquakes with $K_{S} \geq 13.5$ over the period 1962-2018 this parameter showed that its reliability $R=0.83$ (abnormal values $\xi_{P}$ was preceded by $83 \%$ of the earthquakes), the accuracy of $V=0.4$ (40\% of the identified abnormal values $\xi_{P}$ ended with earthquakes), the efficiency of this parameter showed that its reliability $R=0.83$ (abnormal values $\xi_{P}$ was preceded by $83 \%$ of the earthquakes). The efficiency $J_{G}$ in A.A. Gusev was 1.65 (for $J_{G}>1.5$, the predictive sign is considered useful). The efficiency $J_{M}$ in G. M. Molchan was 0.54 , which can be interpreted as a high degree of reliability revealed correlations between identified anomalies of the seismic regime with the parameter $\xi_{P}$ and predicted earthquakes with the energy class range. As a short-term precursor, the complex of ionospheric disturbances is considered: exceeding the current values of the critical frequency $f_{\mathrm{oF}} 2$ the ionospheric F2 layer over the median values in periods of perturbed state of the magnetosphere, $\mathrm{K}$ is the layer, the stratification of the F2 mode ("V"), Es type $\mathrm{r}$. Analysis of prognostic efficiency of the algorithm based on the complex ionospheric disturbances at a given waiting period $T_{\mathrm{w}}=5$ day for earthquakes with $K_{S} \geq 13.5$ over the period 2013-2018 in the spring and autumn seasons, it showed that its reliability $R=0.76$ (ionospheric precursors preceded $76 \%$ of earthquakes), the reliability $V=0.17$ (17\% of identified ionospheric disturbances ended in earthquakes), the efficiency of $J_{G}$ according to A. A. Gusev was 2.01, the efficiency of $J_{M}$ according to G. M. Molchan was 0.38 .

Joint analysis of the parameter $\xi_{P}$ and ionospheric precursors before the occurrence of 25 seismic events $K_{S} \geq 13.5$ showed that 14 of them occurred in the waiting area $S_{\text {exp }}$ and the probability of their occurrence was $P\left(D_{1} \mid E\right) \geq 0.7$, at the same time, a complex of ionospheric disturbances was identified in the time interval up to five days prior to the occurrence of these seismic events.

The work was partially supported by the Integrated program of fundamental scientific research of the far Eastern branch of the Russian Academy of Sciences "far East" for 20182020: project 18-5-095 "Development of new methods of integrated geophysical monitoring for the prediction of strong tectonic earthquakes and prevention of accidents in industrial power systems (Section 1)".

\section{References}

[1] V.V.Bogdanov, A.V.Kaysin, A.V. Pavlov, A.L Polyukhova, S.M.Dushkina, O.A.Gasheva Sil'nye zemletrjasenij 2013 (Novaya Kniga, Petropvlovsk-Kamchatsky, 2014)127-135

[2] V.V. Bogdanov, A.V. Kaisin, A.V. Pavlov, A.L. Polyukhova, C.-V. Meister, J. Physics and Chemistry of the Earth 98, 154-160 (2017)

[3] V.V. Bogdanov, Doklady Akademii Nauk 408, 393-397 (2006)

[4] V.V. Bogdanov, A.V. Pavlov, A.L. Polyukhova, J. Volcanol. Seismol. 4 (6), 412-422 (2010)

[5] URL: http://www.emsd.ru/sdis/earthquake/catalogue/catalogue.php

[6] Yu.V. Riznichenko, Issledovaniy po fizike zemletrjasenij (Nauka, Moskva, 1976)

[7] A.D. Zav’yalov, Prognoz zemletrjasenij (Nauka, Moskva, 2006)

[8] V.A. Saltykov, Physics of the Earth 2, 84-96 (2017) 
[9] A.A. Gusev, Seismicity and seismic forecast, properties of the upper mantle and their relation to volcanism on Kamchatka (Nauka, Novosibirsk, 1974) 109-119

[10] G.M. Molchan, Physics of the Earth and Planetary Interiors 61, 84-98 (1990)

[11] V.M. Sorokin, V.M. Chmyrev, O.A. Pokhotelov, V.A. Liperovsky, Kratkij prognoz katastroficheskih zemletrjasenij s pomoshh'ju radiofizicheskih nazemno-kosmicheskih metodov (OIFZ RAS, Moscow, 1998) 64-85

[12] E.V. Liperovskaya, V.V. Bogdanov, P.-F. Biagi, C.-V. Meister, V.A. Liperovsky, M.V. Rodkin, Nat. Hazards Earth Syst. Sci. 9, 1-7 (2009)

[13] E.V. Liperovskaya, V.V. Bogdanov, P.-F. Biagi, C.-V. Meister, V.A.Liperovsky, Nat. Hazards Earth Syst. Sci. 8, 1807-1812 (2011)

[14] V.V. Bogdanov, A.V. Buzevich, A.V. Vinitsky, G.I. Druzhin, A.V. Kuptsov, I.N. Poddelsky, S.E. Smirnov, B.M. Shevtsov, Complex seismological and geophysical research of Kamchatka. To the 25th anniversary of the Kamchatka Experimental-Methodical Seismological Department of the GS RAS (KEMSD GS RAS, Petropavlovsk-Kamchatsky, 2004) 259-278

[15] V.V. Bogdanov, A.V. Pavlov, E3S Web of Conferences 20, 03001 (2017)

[16] V.V. Bogdanov, A.V. Pavlov, E3S Web of Conferences 63, 03001 (2018) 